\title{
DUAL FUNCTIONS AND HEAT EXPANSIONS ${ }^{1}$
}

L. R. BRAGG

1. Introduction. In recent papers, conditions have been established [1], [3], [4] under which an analytic solution of the radial heat equation

$$
\frac{\partial}{\partial t} u(r, t)=\Delta_{\mu} u(r, t), \quad \Delta_{\mu} \equiv D_{r}^{2}+[(\mu-1) / r] D_{r}
$$

has valid series expansions of the form

$$
\sum_{n=0}^{\infty} a_{n} R_{n}^{\mu}(r, t)
$$

$$
\sum_{n=0}^{\infty} b_{n} \tilde{R}_{n}^{\mu}(r, t)
$$

In these, the $R_{n}^{\mu}(r, t)$ denote the radial heat polynomials while the $\widetilde{R}_{n}^{\mu}(r, t)$ denote their corresponding Appell transforms [1]. Throughout this paper we assume that $\mu>1$. The results show that $u(r, t)$ has the representation (1.2a) valid in the time strip $|t|<1 / 4 \sigma$ provided there exists an entire function $\phi\left(r^{2}\right)$, of growth $(1, \sigma)$ in $r^{2}$, such that

$$
u(r, t)=\int_{0}^{\infty} \frac{1}{2 t} r^{1-\mu / 2 \xi \mu / 2} e^{-\left(r^{2}+\xi^{2}\right) / 4 t} I_{\mu / 2-1}\left(\frac{r \xi}{2 t}\right) \phi\left(\xi^{2}\right) d \xi .
$$

Moreover, $u(r, 0)=\phi\left(r^{2}\right)$. Similarly, $u(r, t)$ has the representation (1.2b) in the halfplane $t>\sigma \geqq 0$ provided that $u(r, t)$ satisfies a Huygens principle as well as the integrability condition

$$
\int_{0}^{\infty} \xi^{\mu-1} e^{\xi^{2} / 8 t}|u(\xi, t)| d \xi<\infty \quad \text { for } t>\sigma .
$$

In this case, there exists an entire function $\psi\left(r^{2}\right)$, of growth $(1, \sigma)$ in $r^{2}$, such that

$$
u(r, t)=(2 \pi)^{-\mu / 2} \int_{0}^{\infty} r^{1-\mu / 2 \xi \xi^{\mu / 2}} J_{\mu / 2-1}(r \xi) e^{-\xi^{2} t} \psi\left(\xi^{2}\right) d \xi .
$$

The coefficients in (1.2a) and (1.2b) are given, respectively, by

Received by the editors October 4, 1965.

1 The research on this paper was supported in part by the National Aeronautics and Space Administration Grant Number NSG-544. 


$$
a_{n}=\frac{1}{(2 n) !} \phi^{(2 n)}(0)
$$

(b)

$$
b_{n}=\frac{(-1)^{n}}{4^{n}(2 n) !} \psi^{(2 n)}(0)
$$

$$
n=0,1,2, \cdots
$$

There exist solutions of (1.1) that have expansions of one of these types but not the other.

There remain a number of questions pertaining to these expansions. For example, if a solution of (1.1) is to satisfy the condition $u(r, 0)$ $=\phi\left(r^{2}\right)$, is there a simple test to determine if $u(r, t)$ has the expansion (1.2b) without evaluating (1.3) and validating condition (1.4)? If so, can the coefficients $b_{n}$ in this expansion be determined from the data? It is evident that the data should provide all required information. Next, suppose that the expansion $(1.2 \mathrm{~b})$ converges to an analytic solution of (1.1) for $t>\sigma>0$. Is it possible to continue this solution across the boundary $t=\sigma$ to define an expansion of the form (1.2a)? If not, does there exist an expansion of the form (1.2a) holding in any time strip?

Answers to these and similar questions can be provided by obtaining connections between the dual functions $\phi\left(r^{2}\right)$ and $\psi\left(r^{2}\right)$ defined above. It will be shown in $\$ 2$ that both expansions (1.2) hold if there exists a pair of symmetrical integral relationships between these dual functions. The relationships resemble Hankel transforms. Introducing Laplace transforms, necessary and sufficient conditions are obtained (in §3) in order that an entire function $\phi\left(r^{2}\right)$ define an entire dual function $\psi\left(r^{2}\right)$. The dual of $\phi\left(r^{2}\right)$ (or $\psi\left(r^{2}\right)$ ) may fail to exist or else exist only as a distribution. In the former case, we refer to $t=1 / 4 \sigma$ as a natural boundary of $u(r, t)$. In the latter case, the integrals (1.3) and (1.5) lead to the same series expansions. Examples are provided to illustrate theorems. The connection between the above problem and D. V. Widder's studies on the resemblance between classical function theory and classes of solutions of the standard heat equation [7] is evident.

2. Symmetrical integral relationships. Let $\phi\left(r^{2}\right)$ and $\psi\left(r^{2}\right)$ be entire functions that satisfy the following pair of relations

(a) $\psi\left(r^{2}\right)=(2 \pi)^{\mu / 2} r^{1-\mu / 2} \int_{0}^{\infty} x^{\mu / 2} J_{\mu / 2-1}(r x) \phi\left(x^{2}\right) d x$,

(b) $\phi\left(r^{2}\right)=(2 \pi)^{-\mu / 2} r^{1-\mu / 2} \int_{0}^{\infty} x^{\mu / 2} J_{\mu / 2-1}(r x) \psi\left(x^{2}\right) d x$. 
Throughout this section, we assume that $x^{\mu / 2} \phi\left(x^{2}\right)$ and $x^{\mu / 2} \psi\left(x^{2}\right)$ $\in L^{1}(0, \infty)$. Under these conditions, we have

TheOREM 2.1. Let $\psi\left(r^{2}\right)$ be defined by (2.1a). Then the integral (1.5) reduces to the integral (1.3).

Proof. Upon substituting (2.1a) into (1.5), we find

$$
\begin{aligned}
(2 \pi)^{-\mu / 2} & \int_{0}^{\infty} r^{1-\mu / 2} \xi^{\mu / 2} J_{\mu / 2-1}(r \xi) e^{-\xi^{2} t} \psi\left(\xi^{2}\right) d \xi \\
& =\int_{0}^{\infty} r^{1-\mu / 2 \xi / 2} J_{\mu / 2-1}(r \xi) e^{-\xi^{2} t}\left(\xi^{1-\mu / 2} \int_{0}^{\infty} x^{\mu / 2} J_{\mu / 2-1}(x \xi) \phi\left(x^{2}\right) d x\right) d \xi \\
& =r^{1-\mu / 2} \int_{0}^{\infty} x^{\mu / 2} \phi\left(x^{2}\right)\left\{\int_{0}^{\infty} \xi J_{\mu / 2-1}(r \xi) J_{\mu / 2-1}(x \xi) e^{-\xi^{2} t} d \xi\right\} d x \\
& =r^{1-\mu / 2} \int_{0}^{\infty} x^{\mu / 2} \phi\left(x^{2}\right)\left\{\frac{1}{2 t} e^{-\left(r^{2}+x^{2}\right) / 4 t} I_{\mu / 2-1}\left(\frac{r x}{2 t}\right)\right\} d x .
\end{aligned}
$$

The last member of this is precisely (1.3). The interchange of orders of integration from the second to the third member follows from the absolute integrability of $x^{\mu / 2} \phi\left(x^{2}\right)$. The evaluation of the inner integral in the third member is given in [5, p. 29, second formula].

By a similar argument, we obtain

TheOREM 2.2. Let $\phi\left(r^{2}\right)$ be defined by (2.1b) where $x^{\mu / 2} \psi\left(x^{2}\right)$ $\in L^{1}(0, \infty)$. Then the integral (1.3) reduces to the integral (1.5).

From these theorems, we see that a pair of functions related by (2.1a) or (2.1b) define a solution function of (1.1) that has expansions of both types indicated in (1.2). The regions in which these different expansions are valid need not be adjacent.

EXAmple 1. Select $\mu=2$ and $\phi\left(r^{2}\right)=e^{-r^{2}}+e^{-2 r^{2}}$. Then $\psi\left(r^{2}\right)$ $=\pi / 2\left\{e^{-r^{2} / 4}+\frac{1}{2} e^{-r^{2} / 8}\right\}[5$, p. 137]. It follows that both types of expansions (1.2) hold for the corresponding $u(r, t)$. The first expansion holds for $|t|<\frac{1}{8}$ while the second expansion is valid for $t>\frac{1}{4}$ (Theorem 5.1 and Theorem 7.3 of [1]).

3. Laplace transform criterion. The integrability requirements on $\psi\left(x^{2}\right)$ and $\phi\left(x^{2}\right)$ in $\S 2$ are more stringent than are necessary to obtain both types of expansions (1.2). The function $\phi\left(r^{2}\right)$ may have an oscillatory character and lead to a conditionally convergent integral in (2.1a). For the purpose of treating such functions, it is convenient to relate $\phi$ and $\psi$ by Laplace transforms. Following the formulation in $[2$, Theorem 2.2$]$, we have 
THEOREM 3.1. Let

$$
T_{\mu}(s)=\int_{0}^{\infty} e^{-x s} x^{\mu / 2-1} \phi(x) d x .
$$

Then

$$
\psi\left(r^{2}\right)=\frac{1}{4}(2 \pi)^{\mu / 2} r^{2-\mu} L_{p}^{-1}\left\{p^{-\mu / 2} T_{\mu}(1 / p)\right\},
$$

in which the variable in this inverse Laplace transform is replaced by $r^{2} / 4$.

By introducing the method of proof of Theorem (14.a), p. 96 of [6], we readily obtain:

LEMMA 3.1. Let

$$
f(s)=\sum_{n=0}^{\infty} a_{n} \frac{\Gamma(\nu+n+1)}{s^{n+1}} \quad \text { for }|s|>c
$$

with $\nu>-1$ and $c$ a positive constant. Then $s^{-v} f(s)=\int_{0}^{\infty} e^{-s x} x^{\nu} \phi(x) d x$ where $\phi(x)$ is the entire function $\sum_{n=0}^{\infty} a_{n} x^{n}$.

If we make use of Stirling's formula, it follows that the above series for $f(s)$ converges for $|s|>c$ if and only if the series $\sum_{n=0}^{\infty}\left(a_{n} n ! / s^{n+1}\right)$ converges for $|s|>c$. Upon combining this observation with Corollary (14.c), p. 96 of [6] and using Theorem 3.1, we find

TheOREM 3.2. Let $\mu>1$ and let $\phi\left(r^{2}\right)$ be an entire function of $r^{2}$ of order $O\left(e^{\epsilon r^{2}}\right)$ as $r \rightarrow \infty$ for arbitrary $\epsilon>0$. Let $F(s)=\int_{0}^{\infty} e^{-s x} \phi(x) d x$. Then the dual function $\psi\left(r^{2}\right)$ of $\phi\left(r^{2}\right)$ is entire in $r^{2}$ of order $O\left(e^{*} r^{2}\right)$ for arbitrary $\epsilon^{*}>0$ as $r \rightarrow \infty$ if and only if $F(s)$ and $s^{\mu / 2-2} F(1 / s)$ are both entire functions of $1 / s$ for $|s|>c$.

This theorem shows that the dual of the entire function $\phi\left(r^{2}\right)$ is entire if the function $s^{\mu / 2-1} T_{\mu}(s)$ in Theorem 3.1 involves, at worst, poles or branch poles. These can only lie in the left halfplane or along the imaginary axis with $\operatorname{Im}(s) \neq 0$. Under these conditions, the corresponding $u(r, t)$ has expansions of both types indicated in (1.2). This also shows that if one of the representations (1.2a) or (1.2b) is given, then the corresponding $\phi\left(r^{2}\right)$ or $\psi\left(r^{2}\right)$ can be determined. The existence of the other type of series representation then reduces to the existence of an entire dual function. Note that if $T_{\mu}(s)$ fails to exist for $0<\operatorname{Re}(s)<a$, then $u(r, t)$ corresponding to $\phi\left(r^{2}\right)$ has a natural boundary at $t=1 / 4 a$. This follows from Theorem 2.1 of [2]. If $\phi\left(r^{2}\right)$ and $\psi\left(r^{2}\right)$ are interchanged in Theorem 3.1 and $(2 \pi)^{\mu / 2}$ is replaced by $(2 \pi)^{-\mu / 2}$ in (3.2), we obtain an analogous theorem. However, in this 
situation if $T_{\mu}(s)$ fails to exist for $0<\operatorname{Re}(s)<a$, then $u(r, t)$ corresponding to $\psi\left(r^{2}\right)$ has a natural boundary at $t=a$ (Theorem 2.2 of $[2])$.

EXAMPLE 2. Let $\mu=2$ and $\phi\left(r^{2}\right)=\sin r^{2}$. By Theorem 3.1, we have $\psi\left(r^{2}\right)=\pi \cos \left(r^{2} / 4\right)$. From (1.6), we obtain $a_{2 n}=0, a_{2 n+1}=(-1)^{n} /(2 n+1)$ ! and $b_{2 n}=(-1)^{n} \pi / 4^{4 n}(2 n) !, b_{2 n+1}=0$ as the coefficients in (1.2a) and (1.2b). In this example,

$$
u(r, t)=\left(1+16 t^{2}\right)^{-1} e^{-4 r^{2} t /\left(1+16 t^{2}\right)}\left\{\sin \frac{r^{2}}{1+16 t^{2}}+4 t \cos \frac{r^{2}}{1+16 t^{2}}\right\} .
$$

Example 3. Let $\mu>1$ and consider the expansion

$$
\sum_{n=0}^{\infty}(-1)^{n} / \Gamma(n+1 / 2) \widetilde{R}_{n}^{\mu}(r, t) .
$$

From (1.6b) we find

$$
\psi\left(r^{2}\right)=\sum_{n=0}^{\infty}\left(4 r^{2}\right)^{n} / \Gamma(n+1 / 2)=\pi^{-1 / 2}+2 r e^{4 r^{2}} \operatorname{erf}(2 r) .
$$

As $r$ tends to infinity, $\psi\left(r^{2}\right)$ behaves as $\pi^{-1 / 2}+2 r e^{4 r^{2}}$. It is clear from Theorem 3.1 (with $\phi$ and $\psi$ interchanged) that there exists no corresponding dual function $\phi\left(r^{2}\right)$. Since the integral (3.1) fails to exist for $s<4$, the solution $u(r, t)$ defined by the given expansion has a natural boundary at $t=4$.

4. Generalized dual functions. The dual of an entire function $\phi\left(r^{2}\right)$ may fail to be entire either because $T_{\mu}(s)$ in Theorem 3.1 does not exist for some $s$ with $\operatorname{Re}(s)>0$ or because the conditions of Theorem 3.2 are not satisfied. If, however, $\phi\left(r^{2}\right)$ is the product of a polynomial in $r^{2}$ by a bounded entire function of $r^{2}$, a simple calculation in Theorem 3.1 shows that $\left|p^{-\mu / 2} T_{\mu}\left(p^{-1}\right)\right| \leqq M|p|^{[\mu / 2]+1}$ for $M$ a positive constant and $\operatorname{Re}\{p\}$ sufficiently large. Under these circumstances, $p^{-\mu / 2} T_{\mu}\left(p^{-1}\right)$ is the Laplace transform of a distribution bounded on the left at $r=0[8$, p. 236]. Depending upon the choice of $\phi\left(r^{2}\right)$, the support of the distribution may lie to the right of $r=0$. The function $\phi\left(r^{2}\right)$ can grow more rapidly than a polynomial but less rapidly than a function bounded by $M e^{\alpha r^{\beta}}$, for $\alpha>0,0<\beta<1$, and still give rise to a generalized dual $\psi\left(r^{2}\right)$. For the most general conditions on $T_{\mu}(s)$ in order that $\psi\left(r^{2}\right)$ be a distribution, see $[8$, p. 258]. The two-sided Laplace transform is needed to treat this case.

In order that $u(r, t)$, corresponding to an entire $\phi\left(r^{2}\right)$, have an expansion in the set $\left\{\tilde{R}_{j}^{\mu}(r, t)\right\}_{j=0}^{\infty}$, it is necessary that the dual of 
$\phi\left(r^{2}\right)$ be entire in $r^{2}$. The above discussion shows that if the dual of $\phi\left(r^{2}\right)$ is a generalized function that is not entire, then $u(r, t)$ has expansions in the set $\left\{R_{n}^{\mu}(r, t)\right\}_{n=0}^{\infty}$ valid for all $r \geqq 0$ and all $t$. Since the relation between $\phi\left(r^{2}\right)$ and $\psi\left(r^{2}\right)$ is symmetrical, we see that if the dual of $\psi\left(r^{2}\right)$ is a nonentire generalized function, then $u(r, t)$ has valid expansions in the set $\left\{\tilde{R}_{n}^{\mu}(r, t)\right\}_{n=0}^{\infty}$ for $t>\sigma \geqq 0, \sigma$ a constant. The generalized dual of $\psi\left(r^{2}\right)$ acts as a potential function concentrated along the $r$-axis (see [2, Theorem 3.3]).

EXAmple 4. Select $\mu=2$ and $\phi\left(r^{2}\right)=\delta\left(r^{2}-a^{2}\right), a>0$. From Theorem 3.1, we find that $\psi\left(r^{2}\right)=(\pi / 2) J_{0}(a r)$. Using (1.6), we obtain $b_{n}=(\pi / 2) a^{2 n} / 2^{4 n}(n !)^{2}$ for the coefficients in (1.2b). That expansion holds for $t>0$. The corresponding $u(r, t)$ in this case is $(8 t)^{-1} e^{-\left(r^{2}+a^{2}\right) / 4 t} I_{0}(a r / 2 t)$.

\section{REFERENCES}

1. L. R. Bragg, The radial heat polynomials and related functions, Trans. Amer. Math. Soc. 119 (1965), 270-290.

2. - The radial heat equation and Laplace transforms, J. SIAM Appl. Math. 14 (1966), 986-993.

3. D. T. Haimo, Functions with the Huygens property, Bull. Amer. Math. Soc. 71 (1965), 528-532.

4. - Expansions in terms of generalized heat polynomials and of their Appell transforms, J. Math. Mech. 15 (1966), 735-758.

5. W. Magnus and F. Oberhettinger, Formulas and theorems for functions of mathematical physics, Chelsea, New York, 1949.

6. D. V. Widder, The Laplace transform, Princeton Univ. Press, Princeton, N. J., 1946.

7. - Some analogies from classical analysis in the theory of heat conduction, Arch. Rational Mech. Anal. 21 (1966), 108-120.

8. A. H. Zemanian, Distribution theory and transform analysis, McGraw-Hill, New York, 1965.

OAKLAND UNIVERSITY 